\title{
Quantitative MRI can detect subclinical disease progression in muscular dystrophy
}

\author{
Arne Fischmann • Patricia Hafner • \\ Susanne Fasler • Monika Gloor • Oliver Bieri • \\ Ueli Studler · Dirk Fischer
}

Received: 14 August 2011/Revised: 21 December 2011/Accepted: 21 December 2011/Published online: 2 February 2012

(c) Springer-Verlag 2012

\begin{abstract}
Oculopharyngeal muscular dystrophy (OPMD) is a rare autosomal dominant muscular dystrophy with late onset and slow progression. The aim of this study was to compare different methods of quantitative MRI in the follow-up of OPMD to semiquantitative evaluation of MRI images and to functional parameters. We examined 8 patients with genetically confirmed OPMD and 5 healthy volunteers twice at an interval of 13 months. Motor function measurements (MFM) were assessed. Imaging at $1.5 \mathrm{~T}$ (Siemens Magnetom Avanto) comprised two axial slice groups at the largest diameter of thigh and calf and included T1w TSE, 2-point Dixon for muscular fat fraction (MFF) and a multi-contrast TSE sequence to calculate
\end{abstract}

Electronic supplementary material The online version of this article (doi:10.1007/s00415-011-6393-2) contains supplementary material, which is available to authorized users.

A. Fischmann $(\bowtie)$

Department for Diagnostic and Interventional Neuroradiology, Institute of Radiology, University of Basel Hospital,

Petersgraben 4, 4031 Basel, Switzerland

e-mail: arne.fischmann@unibas.ch

P. Hafner · S. Fasler $\cdot$ D. Fischer

Department of Neurology, University of Basel Hospital,

Basel, Switzerland

M. Gloor · O. Bieri

Division of Radiological Physics, Institute of Radiology,

University of Basel Hospital, Basel, Switzerland

U. Studler

Musculoskeletal Radiology, Institute of Radiology,

University of Basel Hospital, Basel, Switzerland

D. Fischer

Department of Neuropaediatrics,

University of Basel Children's Hospital, Basel, Switzerland quantitative T2 values. T1 images were analyzed using Fischer's semiquantitative 5-point (0-4) scale. MFM and visual scores showed no significant difference over the study period. Overall $\mathrm{T} 2$ values increased in patients over the study period from 49.4 to $51.6 \mathrm{~ms}$, MFF increased from 19.2 to $20.7 \%$. Neither T2 values nor MFF increased in controls. Changes in T2 correlated with the time interval between examinations $\left(r^{2}=0.42\right)$. In this small pilot trial, it was shown that quantitative muscle MRI can detect subclinical changes in patients with OPMD. Quantitative MRI might, therefore, be a useful tool for monitoring disease progression in future therapeutic trials.

Keywords MRI - Neuromuscular disease · OPMD . Follow-up

\section{Introduction}

Oculopharyngeal muscular dystrophy (OPMD) is a rare autosomal dominant muscular dystrophy with late onset and slow progression, caused by a repeat expansion in the PABPN-1 (polyadenlyate binding protein-1) gene. Disease prevalence worldwide is $1: 100,000$, but is much higher in certain communities, e.g., French Canadians and Bukhara Jews. Symptoms include initial ptosis and difficulties swallowing as well as later proximal weakness [1]. We recently showed that semiquantitative muscular magnetic resonance imaging (MRI) correlates with clinical abilities [2].

Future trials in muscular dystrophies require validated objective outcome measures. One main objective of the international Treat-NMD-Network is to develop such standardized protocols [3]. Unfortunately, even validated clinical scales are influenced by fatigue, concentration, cooperation, and limited inter- and intrarater repeatability. 
In contrast, objective and quantitative biomarkers to assess the severity and progression of muscle degeneration are still largely lacking, with quantitative MRI being a very promising method. MRI has some important advantages: it is noninvasive, time-sparing, includes all muscles of an anatomic region and identical fat/water phantoms can be used for calibration in different multicenter trial sites. Multiple quantitative methods to evaluate muscular fat content have been evaluated $[4,5]$. But only a few trials describe follow-up findings of MRI in neuromuscular disorders $[4,6]$.

The aim of this study was to compare different objective quantitative and semiquantitative MRI methods to validated clinical parameters in the follow-up of OPMD.

\section{Materials and methods}

Eight patients with genetically confirmed oculopharyngeal muscular dystrophy were recruited (age 41-76 years, see Table 1). All patients were heterozygous carriers of a $(\mathrm{GCN})_{13}$ allele with the exception of patient three who had a $(\mathrm{GCN})_{15}$ allele. The clinical data and initial MRI findings were previously reported [2]. In addition, 5 healthy individuals (aged $28 \pm 6$ years) were examined twice at an interval of 13 months to establish stability of quantitative measurements. All measurements were approved by the local ethics committee (EKBB No. 83/08) and all participants gave written informed consent.

Physical assessment was performed prior to imaging in all but 2 patients, who did not wish to participate in this part of the study, using the motor function measurement
(MFM) scale, performed by a physiotherapist trained in MFM scoring. This scale evaluates standing position and transfers, axial and proximal motor function as well as distal motor function. Subset scores for each dimension and a total score are expressed in percentage points. A healthy volunteer achieves $100 \%$ in all dimensions, while a score below $60 \%$ usually indicates loss of ambulation [7]. It has been shown that this scale has a high interrater reliability, does not require any special equipment, and is well tolerated by patients [8].

All imaging was performed on a 1.5-T scanner (Magnetom Avanto, Siemens, Erlangen, Germany) with a 16-element matrix leg angiography coil. Imaging parameters were identical to the sequences reported in a previous publication [5].

In short, two axial slice groups were centered at $14.5 \mathrm{~cm}$ below and $17.5 \mathrm{~cm}$ above the knee joint, respectively, to include the largest diameter of the thigh and the lower leg. An imaging matrix of $384 \times 384$ and a field of view of $400 \times 400 \mathrm{~mm}^{2}$ were used, resulting in a $1 \mathrm{~mm}$ in-plane resolution with $3 \mathrm{~mm}$ slice thickness.

Imaging sequences included a standard T1-weighted turbo spin echo (TSE) sequence ( 30 slices, $\mathrm{TR}=457 \mathrm{~ms}$, $\mathrm{TE}=7.5 \mathrm{~ms}$ ) for anatomical reference and visual evaluation of fatty replacement, three-dimensional (3D) gradient echo sequence with two different echo times for in-phase and opposed-phase imaging (3D, 30 slices, TR $=20 \mathrm{~ms}$, $\mathrm{TE} 1=2.38$, TE2 $=4.76 \mathrm{~ms}$, flip angle $=25^{\circ}$ ). T2 quantification was based on a multicontrast TSE sequence (3 slices, $\mathrm{TR}=1,330 \mathrm{~ms}, \mathrm{TE}=10,20,30, \ldots, 320 \mathrm{~ms}-31$ echoes). The entire protocol was completed within 20:44 min.

Table 1 Motor function measurements (MFM) and visual evaluation of fatty replacement for all patients. The youngest patient (patient 6) presented without symptoms of OPMD

\begin{tabular}{|c|c|c|c|c|c|c|c|c|c|c|}
\hline $\begin{array}{l}\text { Patient } \\
\text { No }\end{array}$ & Age & $\begin{array}{l}\text { Age } \\
\text { at } \\
\text { onset }\end{array}$ & Sex & $\begin{array}{l}\text { Interval } \\
\text { between } \\
\text { scans }\end{array}$ & $\begin{array}{l}\text { Initial } \\
\text { evaluation } \\
\text { MFM av (\%) }\end{array}$ & $\begin{array}{l}\text { Repeat } \\
\text { evaluation } \\
\text { MFM av (\%) }\end{array}$ & $\begin{array}{l}\text { Average } \\
\text { visual Reader } \\
1 \text { initial }\end{array}$ & $\begin{array}{l}\text { Average } \\
\text { visual Reader } \\
1 \text { repeat }\end{array}$ & $\begin{array}{l}\text { Average } \\
\text { visual Reader } \\
2 \text { initial }\end{array}$ & $\begin{array}{l}\text { Average } \\
\text { visual Reader } \\
2 \text { repeat }\end{array}$ \\
\hline 1 & 75 & 40 & $\mathrm{~F}$ & 11.2 & 97.9 & 99.0 & 0.31 & 0.36 & 1.10 & 1.10 \\
\hline 2 & 69 & 45 & M & 12.2 & 84.4 & 83.3 & 2.52 & 2.52 & 2.47 & 2.47 \\
\hline 3 & 59 & 49 & F & 12.2 & 96.9 & 99.0 & 1.05 & 1.05 & 1.32 & 1.32 \\
\hline 4 & 61 & 55 & $\mathrm{~F}$ & 16.3 & 91.7 & 92.8 & 2.0 & 2.0 & 1.42 & 1.42 \\
\hline 5 & 63 & 50 & $\mathrm{~F}$ & 16.3 & 93 & 96.9 & 1.05 & 1.0 & 2 & 2 \\
\hline 6 & 41 & n.a. & M & 16.3 & 100 & 100 & 0.25 & 0.21 & 1.25 & 1.25 \\
\hline 7 & 54 & - & $\mathrm{F}$ & 10.1 & n.a. & n.a. & 0.47 & 0.47 & 1.79 & 1.79 \\
\hline 8 & 69 & 50 & $\mathrm{~F}$ & 8.5 & n.a. & n.a. & 0.26 & 0.26 & 1.42 & 1.42 \\
\hline Mean & & & & 13.0 & 93.98 & 95.2 & 0.91 & 0.91 & 1.64 & 1.64 \\
\hline SD & & & & & 5.63 & 6.3 & 0.78 & 0.78 & 0.50 & 0.50 \\
\hline
\end{tabular}

Visual scores by reader 1 showed minimal differences (patients 1, 5, and 6) between initial and repeat scans. Reader 2 detected no differences between initial and repeat scans

SD Standard deviation, n.a. not applicable 
Repeat imaging in patients was performed after an average of 13 months (8.5-16.3 month), due to restricted patient availability and a large recruitment area. All volunteers were rescanned after 13 months (mean 13 months, SD 0.1 months).

Two radiologists experienced in neuromuscular imaging, blinded to clinical findings, evaluated the images as previously described [2] using a visual 5-point scale [9]. Table 2 lists all the evaluated muscles. The mean fat fraction (MFF) was calculated using a 2-point Dixon (2PD) reconstruction method. $\mathrm{T} 2$ relaxation times were calculated from a pixel-by-pixel mono-exponential least-squares fit to the signal at echo times ranging from $20-320 \mathrm{~ms}$ as described previously [5]. Calculations were made based on regions of interest (ROI) encompassing the cross-sectional area of the whole muscle on three slices, avoiding chemical shift artifacts, as given by the T2 TSE sequence [5]. In addition, ROI positioning was confirmed on the fat, water, and T2 maps (see Fig. 1).

Imaging scores and T2 values were analyzed with averaged values of both legs due to symmetrical involvement. Arithmetic mean of the visual scores from the thigh, lower leg, and whole leg were calculated and compared to clinical assessment given by the MFM scale. Mean MFF from 2PD and mean T2 values of each ROI were averaged across both legs and across the three slices given by the T2-weighted images [5]. Differences between initial and follow-up images were compared using a two-sided $t$ test. Correlations of quantitative fat content, clinical and MRI scores as well as correlations with age and interval between imaging were calculated as Spearman's rank coefficient with JMP 8.0.2 (SAS Institute, Cary, NC, USA).

\section{Results}

Follow-up average MFM scores showed no significant difference $(p=0.18)$ of functional abilities compared to the initial examination (see Table 1). Examples of fatty replacement are shown in Fig. 2. MFM scores decreased with increasing fat fraction $(r=-0.98 /-0.92$ for initial/ repeat) and with increasing fatty replacement on visual evaluation ( $r=-0.98$ for initial and repeat), as well as with increasing T2 values ( $r=-0.94 /-0.90$ for initial/ repeat) (see additional online material E1). This negative correlation was significant for all analyses $(p<0.01)$.

Semiquantitative visual scores showed no relevant change over the study period with an average change per patient of $0.0(-0.05$ to 0.05$)$. When visual changes were observed, these were limited to a maximum of one muscle per patient.
Table 2 Quantitative T2 measurements and MFF over all patients per muscle

$p$ is given for the two-sided $t$ test

* Significance at a $95 \%$ level

\begin{tabular}{|c|c|c|c|c|c|c|}
\hline Muscle & $\begin{array}{l}\mathrm{T} 2 \text { (in } \mathrm{ms} \text { ) } \\
\text { initial }\end{array}$ & $\begin{array}{l}\mathrm{T} 2 \text { (in ms) } \\
\text { follow-up }\end{array}$ & $p$ & $\begin{array}{l}\text { Fat fraction } \\
\text { (in \%) initial }\end{array}$ & $\begin{array}{l}\text { Fat fraction } \\
\text { (in } \% \text { ) follow up }\end{array}$ & $p$ \\
\hline Rectus femoris & 31.8 & 32.1 & 0.76 & 7.4 & 6.1 & 0.9 \\
\hline Vastus intermedius & 41.9 & 43.8 & 0.11 & 13.4 & 14.6 & 0.18 \\
\hline Vastus lateralis & 36.6 & 37.3 & 0.34 & 9.1 & 9.2 & 0.8 \\
\hline Vastus medialis & 40.1 & 41.5 & 0.07 & 10.2 & 11.1 & 0.24 \\
\hline Long biceps & 56.3 & 58.8 & 0.05 & 27.5 & 31.0 & $0.01 *$ \\
\hline Short biceps & 46.7 & 49.1 & 0.06 & 19.7 & 21.4 & 0.10 \\
\hline Semimembranosus & 58.7 & 61.1 & $<0.01 *$ & 30.6 & 32.9 & $<0.01 *$ \\
\hline Semitendinosus & 50.2 & 52.7 & $0.04 *$ & 24.2 & 26.1 & 0.07 \\
\hline Adductor magnus & 71.7 & 77.8 & $<0.01 *$ & 42.8 & 46.9 & $0.01 *$ \\
\hline Gracilis & 35.8 & 38.2 & 0.07 & 11.8 & 11.7 & 0.59 \\
\hline Sartorius & 43.1 & 45.8 & 0.10 & 16.4 & 17.8 & 0.35 \\
\hline Anterior tibial & 43.8 & 45.8 & 0.14 & 9.8 & 11.6 & $0.04 *$ \\
\hline Digital extensor & 53.3 & 56.1 & $<0.01 *$ & 18.4 & 20.4 & $0.04 *$ \\
\hline Peroneus & 54.0 & 55.9 & 0.05 & 21.2 & 22.1 & 0.38 \\
\hline Posterior tibial & 50.8 & 52.0 & 0.18 & 15.3 & 17.6 & $0.04 *$ \\
\hline Soleus & 64.9 & 66.6 & 0.12 & 29.2 & 32.2 & $0.02 *$ \\
\hline Medial gastroc & 56.3 & 57.4 & 0.44 & 22.3 & 22.4 & 0.90 \\
\hline Lateral gastroc & 53.2 & 57.0 & 0.15 & 15.6 & 18.3 & 0.17 \\
\hline Mean thigh & 46.6 & 48.9 & $<0.001^{*}$ & 19.4 & 20.8 & $<0.001^{*}$ \\
\hline Mean calf & 53.7 & 55.8 & $<0.001^{*}$ & 18.8 & 20.6 & $<0.001^{*}$ \\
\hline Mean all muscles & 49.4 & 51.6 & $<0.001 *$ & 19.2 & 20.7 & $<0.001^{*}$ \\
\hline
\end{tabular}


Fig. 1 Typical fat fraction maps (a, b) and T2 maps (c, d) of the right thigh. ROIs (a, c) superimposed on the images with annotations for the muscle groups examined. The hamstrings are shaded in blue, while the quadriceps femoris is shaded in red. $A M$ adductor magnus, $B B$ biceps femoris short head, $B L$ biceps femoris long head, $G R$ gracilis, $R F$ rectus femoris, $S A$ sartorius, $S M$ semimembranosus, $S T$ semitendinosus, $V I$ vastus intermedius, $V L$ vastus lateralis, $V M$ vastus medialis
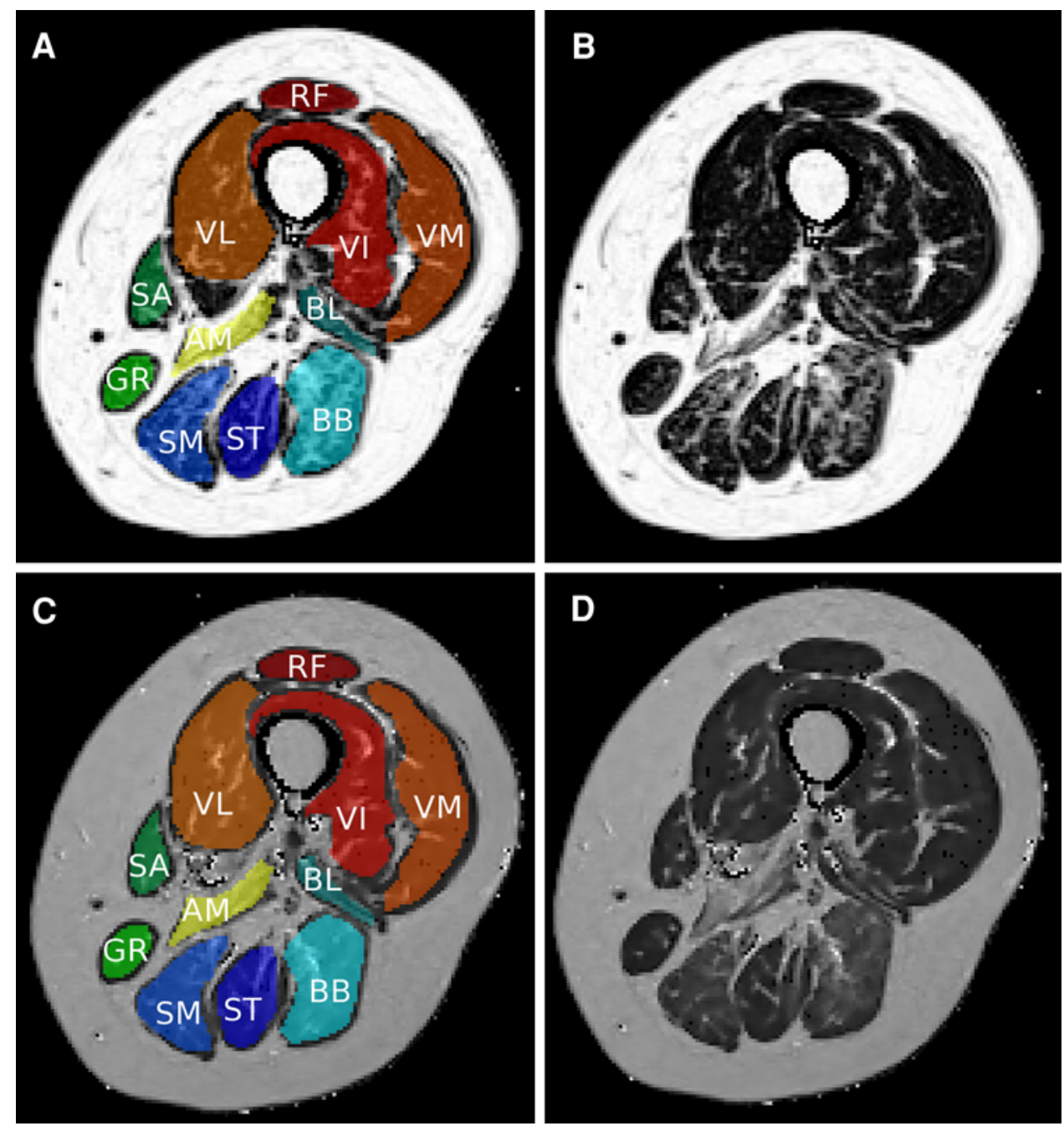

Quantitative measurements showed a significant increase of T2 times and MFF for all patients (Table 2). Controls showed no significant change: T2 was $35.3 \mathrm{~ms}$ (SD 3.83) and $35.2 \mathrm{~ms}$ (SD $4.01 \mathrm{~ms}$ ) at the initial and repeat examinations, respectively $(p=0.44)$, while the corresponding fat fractions were 7.8 (SD 2.6\%) and 7.2\% (SD 2.2\%) for initial and repeat examinations $(p=0.11)$. T2 values in patients were significantly higher compared to controls $(p<0.0001)$ and the change over time was significantly higher in patients $(p=0.01)$ when averaged over all muscles and patients (Fig. 3). However, this was not necessarily the case for each individual subject and muscle (Fig. 4 and Figure E2). Moreover, the change in T2 in patients increased in patients for longer interval between examinations ( $r=0.71$, see Fig. 5).

When averaged over functional-anatomical muscle groups, T2 values in the thigh changed more prominently across the hamstrings (T2 difference $+3.07 \mathrm{~ms}$, $p<0.0001$ ) as compared to the quadriceps (T2 difference $+0.96 \mathrm{~ms}, p=0.04$ ), whereas in the calf, changes in the flexors (soleus and gastrocnemius) were more prominent (T2 difference $+4.0 \mathrm{~ms}$ ) as compared to the extensors (tibialis anterior, extensor digitorum and hallucis longus, peroneus longus) (T2 difference $+1.55 \mathrm{~ms})$. T2 times increased in all muscles, while MFF showed more heterogeneous results and even a decrease in the rectus femoris and gracilis (Table 2). MFF strongly correlated with T2 times with $r^{2}$ of -0.98 and -0.96 at initial scan and follow-up, respectively.

\section{Discussion}

In this study, it has been demonstrated that T2 and MFF quantification could be used to detect subclinical changes in slowly progressive muscular dystrophies such as OMPD. While clinical data and visual analysis of fat content were stable, quantitative measurements, especially T2 measurements showed a constant increase over the study period. This is in accordance with the known history of the disease, 

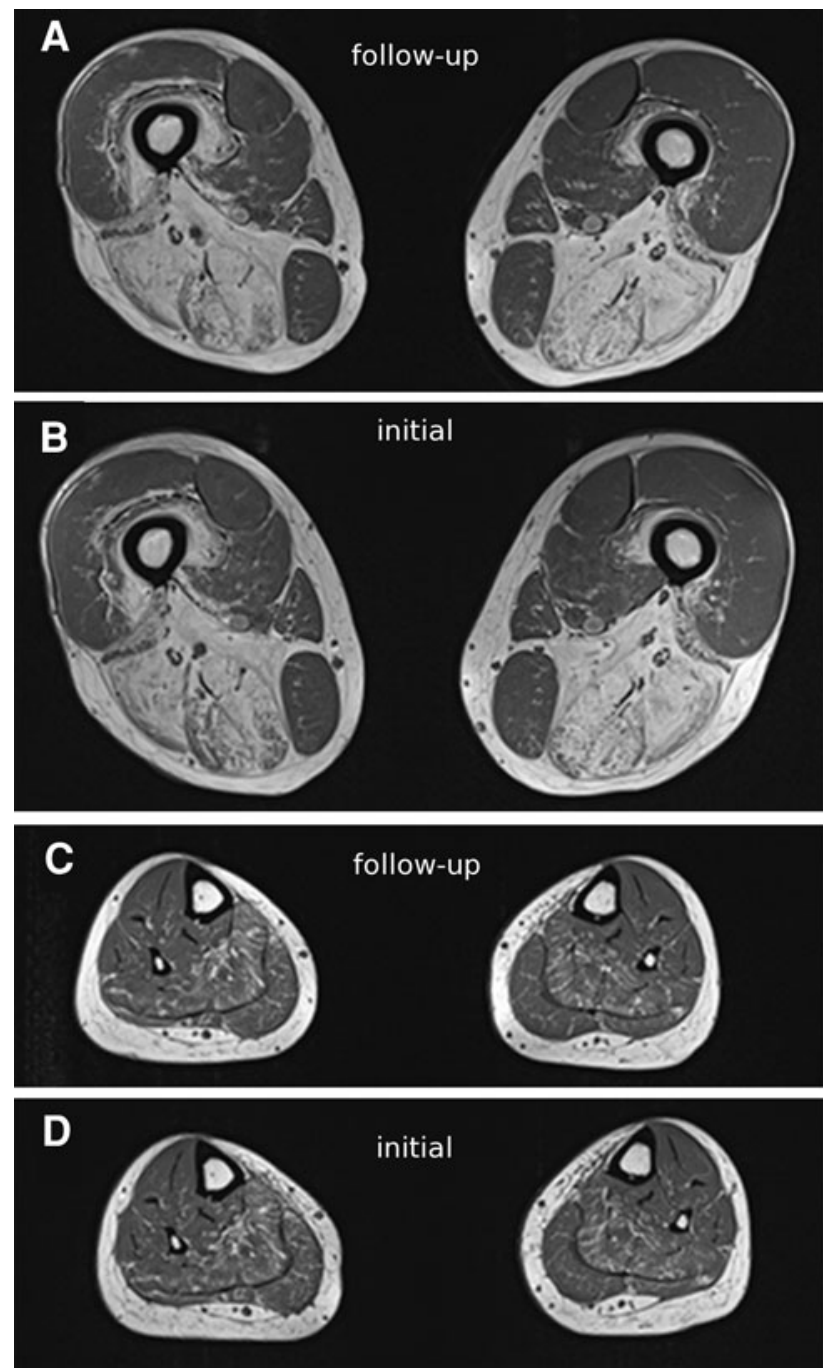

Fig. 2 Images of the thighs $(\mathbf{a}, \mathbf{b})$ from a patient with more advanced involvement of the hamstrings (patient 2) and images from the calves (c, d) of a different patient (patient 3) with moderate involvement predominantly in the soleus. $\mathbf{b}$ and $\mathbf{d}$ are from the initial scan, $\mathbf{a}$ and c from the follow-up study: no difference in the amount of fatty replacement is visible on the scans. Example evaluations by reader $1 / 2$ for the right leg were the following: rectus femoris $=0 / 1$, vastus lateralis $=1 / 2$, vastus intermedius $=3 / 3$, vastus medialis $=1 / 2$, gracilis $=0 / 1$, sartorius $=0 / 1$, semimembranosus $=3 / 3$, semitendinosus $=3 / 3$, short biceps $=2 / 2$, long biceps $=3 / 3$, tibialis anterior $=1 / 2$, peroneus $=1 / 2$, tibialis posterior $=1 / 2$, soleus $=2 / 2$, gastrocnemius lateralis $=1 / 2$, gastrocnemius medialis $=1 / 2$

which is dominated by a very slow progression over long intervals [1], with expected changes in MFM only after an interval of several years. All muscles showed an increase in T2 times, although differences were small, while differences in MFF were more heterogeneous, indicating that $\mathrm{T} 2$ might be more sensitive to slow changes. Even the presymptomatic patient with a known mutation of the PABPN-1 gene had normal MFM scores and no visual signs of fatty infiltration, while T2 times and MFF significantly
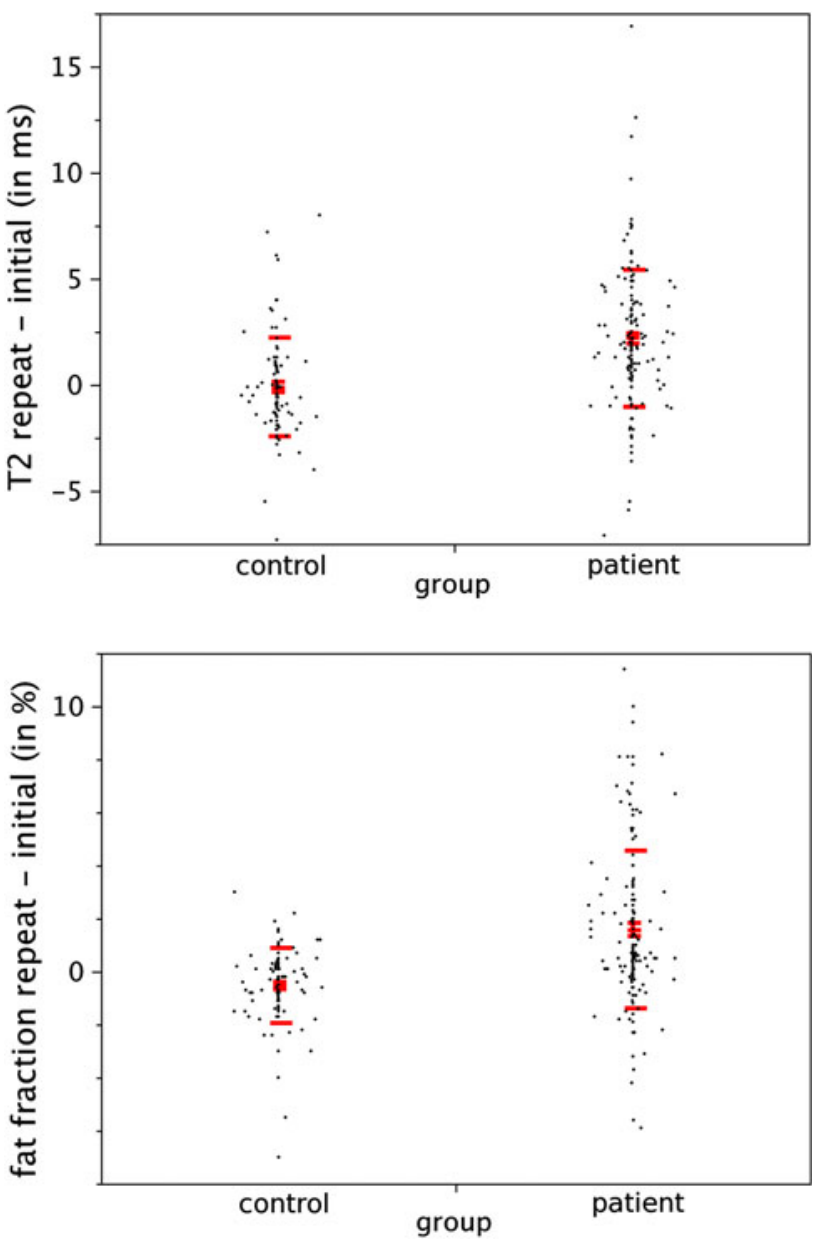

Fig. 3 Comparison of T2 values and MFF between patients and controls on a muscle-by-muscle basis. Red lines indicate mean values, standard deviations, and standard deviations of means

increased over the study period (Fig. 4). This indicates that MRI might be used for monitoring presymptomatic patients, although evidence is currently limited. Whether MRI could also serve as an objective outcome for the assessment in future therapeutic trials needs to be evaluated in future trials with larger cohorts of patients with genetically confirmed OPMD, preferably including groups of younger presymptomatic patients.

Similar to trials in myotonic dystrophy [10] or inclusion body myositis [11], we found a correlation of MRI with functional parameters but not with patient age or disease duration. Kim et al. [4] examined children with Duchenne muscular dystrophy and found increasing $\mathrm{T} 2$ times in 5 patients and decrease in 2 patients after steroid therapy. Bryan et al. [12] found that T2 times correlated with muscle action potential amplitude in patients with ALS. Both studies were in patients with rapidly progressive diseases, while we could show for the first time the use of quantitative follow-up MRI studies in adult muscular dystrophies with slower progression. 


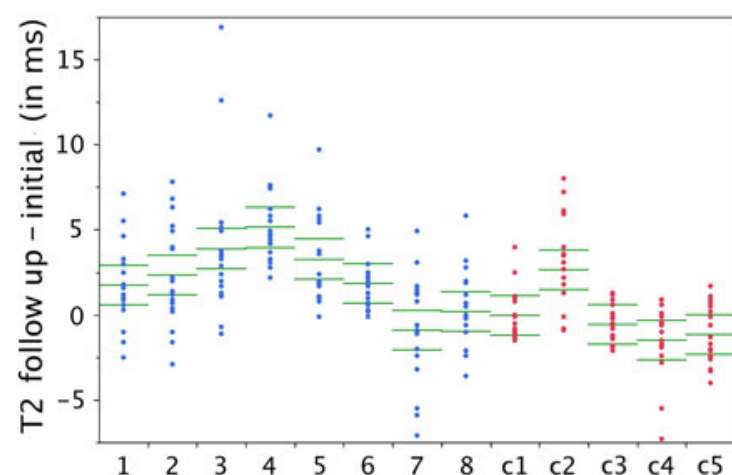

Fig. 4 Comparison of quantitative MRI parameters between patients (1-8 in blue) and controls (c1-c5 in red) plotted as difference between scans per muscle. Green lines indicate mean values \pm one
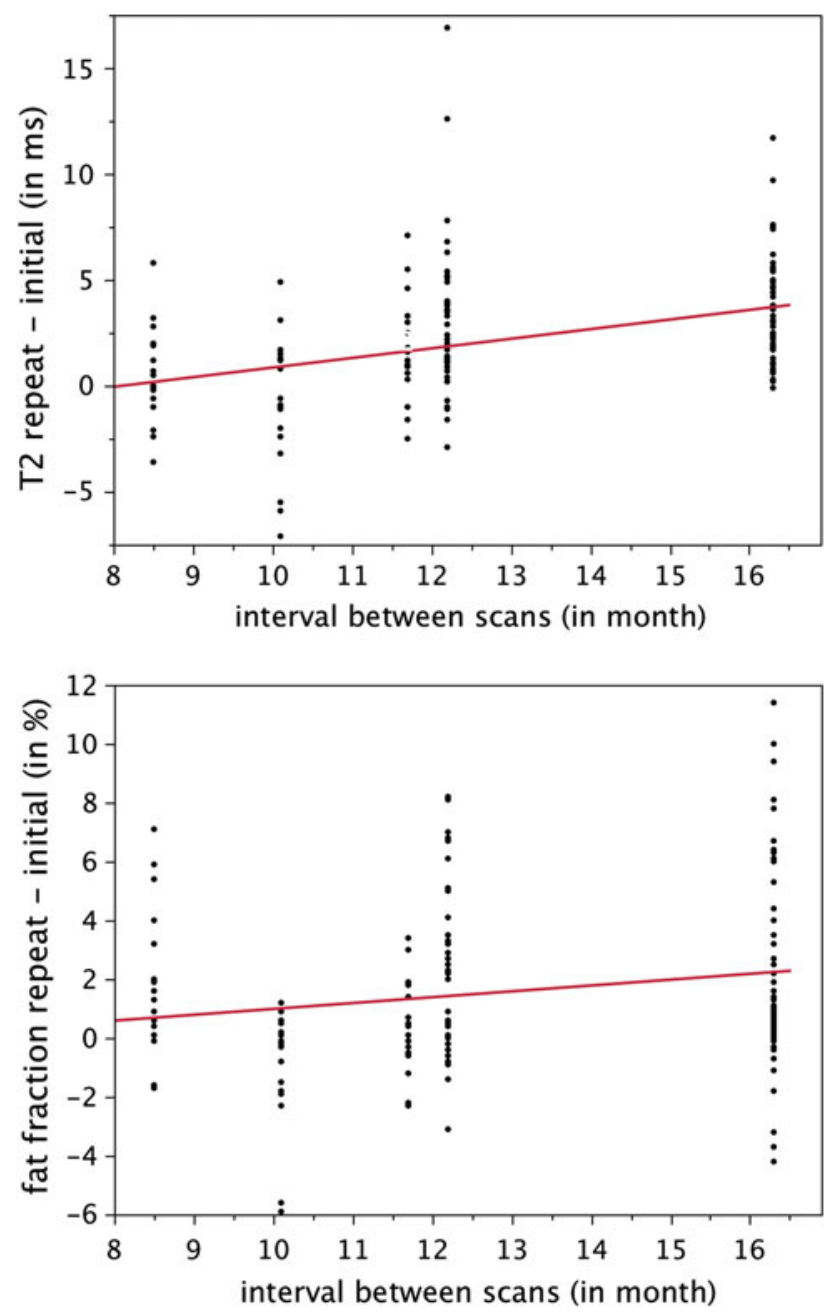

Fig. 5 Changes in T2 values and MFF over time in correlation with scanning interval. The trend line demonstrates the increase in $\mathrm{T} 2$ with increasing interval

Our study has limitations, such as the small number of OPMD patients. We pre-specified statistical evaluations as differences in MFM, T2 and fat fraction between initial and

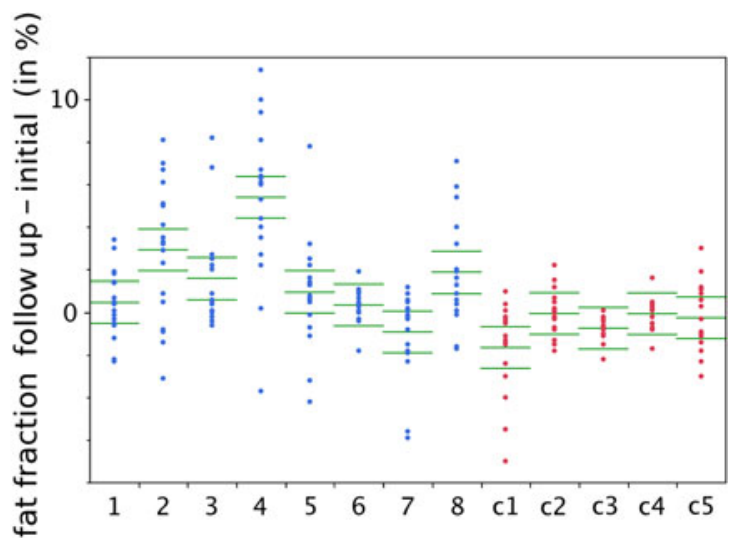

standard deviation. In patients, fat fraction and $\mathrm{T} 2$ values increased in comparison to controls. However, this was not true for all patients or all muscles

repeat exams; these were highly specific. The evaluation of the single muscles in Table 2 was performed to estimate whether specific muscles showed greater changes. The latter evaluations were, however, not corrected for multiple comparisons, $p$ values should, therefore, only be regarded as indications of the direction and size of change.

Another limitation was in the lack of a gold standard for the assessment of the fat infiltration, although MFF on MRI showed excellent agreement to histopathologic results in patients with muscular dystrophy [13]. While fat measurements on muscle biopsy are currently regarded to be the gold standard [14], we were not able to make such correlations by repeated muscle biopsies for ethical reasons. In spectroscopic studies, T2 relaxation measurements correlated with fat content [15]. However, exercise can increase the water content in the intracellular space, which might influence $\mathrm{T} 2$ values [16]. The latter might also be the underlying cause of the variation in T2 values in one volunteer with stable MFF. Furthermore the refusal of 2 patients to participate in MFM evaluation might introduce bias into the clinical correlations.

Another potential limitation lies in potential confounding effects of the 2-point Dixon method. We used an implementation for the 2-point Dixon technique, which accounts for phase errors from magnetic field inhomogeneities. A potential issue of gradient echo based 2-point Dixon imaging is that $\mathrm{T} 1$ and $\mathrm{T} 2 *$ relaxation may cause inaccurate fat and water images [17]. This leads to an overestimation of low MFF and an underestimation of high MFF by the 2-point Dixon method. The 3-point Dixon technique, which is less susceptible to inaccuracies, was not available on Siemens scanners at the time of this study. We also tested other methods of fat quantifications and found them to be less stable compared to the 2-point Dixon method [5]. A determination of absolute $\mathrm{T} 2$ values might also be problematic. The apparent $\mathrm{T} 2$ of lipid protons in 
fast spin-echo MRI is increased [18]. Furthermore, the assumption of a single mono-exponential decay is an oversimplification in the presence of fat [15].

However, these potential issues in 2-point Dixon and T2 imaging have no significant impact on the study outcome, as the main interest was not directed toward absolute values, but rather toward a variation of parameters with disease progression. The latter were verified by the stable results in volunteers over the study period.

In conclusion, we found that quantitative MRI is a sensitive method for the detection of subclinical changes in OPMD: quantitative changes over time can be detected, which do not appear on conventional imaging or in functional measurements like the MFM over the same period. Thus, quantitative MRI might be used as an additional objective tool in the surveillance of progression in muscular dystrophy, but larger trials in OPMD as well as in different muscle diseases need to be performed to verify and validate this finding.

Acknowledgments This study was supported by the Lorenzo Piaggio Foundation, Switzerland. The Institute of Radiology receives an unspecified general grant from Bracco. The sponsor played no role in matters of design, collection, analysis, interpretation of data, and writing the report. The authors would like to thank Tanja Haas for the MRI measurements. We also thank the reviewers for their helpful comments.

Conflicts of interest The authors declare no conflict of interest.

\section{References}

1. Brais B (2009) Oculopharyngeal muscular dystrophy: a polyalanine myopathy. Curr Neurol Neurosci Rep 9:76-82

2. Fischmann A, Gloor M, Fasler S, Haas T, Rodoni Wetzel R, Bieri O, Wetzel S, Heinimann K, Scheffler K, Fischer D (2011) Muscular involvement assessed by MRI correlates to motor function measurement values in oculopharyngeal muscular dystrophy. J Neurol 258:1333-1340. doi:10.1007/s00415-011-5937-9

3. Mercuri E, Mayhew A, Muntoni F, Messina S, Straub V, Van Ommen GJ, Voit T, Bertini E, Bushby K, Network TREATNMDN (2008) Towards harmonisation of outcome measures for DMD and SMA within TREAT-NMD; report of three expert workshops: TREAT-NMD/ENMC workshop on outcome measures, 12th-13th May 2007, Naarden, The Netherlands; TREATNMD workshop on outcome measures in experimental trials for DMD, 30th June-1st July 2007, Naarden, The Netherlands; conjoint Institute of Myology TREAT-NMD meeting on physical activity monitoring in neuromuscular disorders, 11th July 2007, Paris, France. Neuromuscul Disord 18:894-903. doi:10.1016/ j.nmd.2008.07.003

4. Kim HK, Laor T, Horn PS, Wong B (2010) Quantitative assessment of the $\mathrm{T} 2$ relaxation time of the gluteus muscles in children with Duchenne muscular dystrophy: a comparative study before and after steroid treatment. Korean J Radiol 11:304-311. doi:10.3348/kjr.2010.11.3.304

5. Gloor M, Fasler S, Fischmann A, Haas T, Bieri O, Heinimann K, Wetzel SG, Scheffler K, Fischer D (2011) Quantification of fat infiltration in oculopharyngeal muscular dystrophy: comparison of three MR imaging methods. JMRI 33:203-210. doi:10.1002/ jmri.22431

6. Paradas C, Llauger J, Diaz-Manera J, Rojas-García R, De Luna N, Iturriaga C, Márquez C, Usón M, Hankiewicz K, Gallardo E, Illa I (2010) Redefining dysferlinopathy phenotypes based on clinical findings and muscle imaging studies. Neurology 75:316323. doi:10.1212/WNL.0b013e3181ea1564

7. Vuillerot C, Girardot F, Payan C, Fermanian J, Iwaz J, Lattre DE, Berard C (2009) Monitoring changes and predicting loss of ambulation in duchenne muscular dystrophy with the motor function measure. Dev Med Child Neurol. doi:10.1111/j.14698749.2009.03316.x

8. Bérard C, Payan C, Hodgkinson I, Fermanian J, Group MFMCS (2005) A motor function measure for neuromuscular diseases construction and validation study. Neuromuscul Disord 15:463470

9. Fischer D, Kley RA, Strach K, Meyer C, Sommer T, Eger K, Rolfs A, Meyer W, Pou A, Pradas J, Heyer CM, Grossmann A, Huebner A, Kress W, Reimann J, Schröder RJ, Eymard B, Fardeau M, Udd B, Goldfarb L, Vorgerd M, Olivé M (2008) Distinct muscle imaging patterns in myofibrillar myopathies. Neurology 71:758-765. doi:10.1212/01.wnl.0000324927.28817.9b

10. Coté C, Hiba B, Hebert LJ, Vial C, Remec JF, Janier M, Puymirat J (2011) MRI of tibialis anterior skeletal muscle in myotonic dystrophy type 1. Can J Neurol Sci 38:112-118

11. Sinclair C, Morrow J, Fischmann A, Hanna M, Reilly M, Yousry T, Golay X, Thornton J (2011) Skeletal muscle MRI-determined fat fraction and myometric strength in inclusion body myositis and Charcot-Marie-Tooth disease Type 1A. Neuromuscul Disord 21:S5. doi:10.1016/S0960-8966(11)70014-1

12. Bryan WW, Reisch JS, McDonald G, Herbelin LL, Barohn RJ, Fleckenstein JL (1998) Magnetic resonance imaging of muscle in amyotrophic lateral sclerosis. Neurology 51:110-113

13. Gaeta M, Scribano E, Mileto A, Mazziotti S, Rodolico C, Toscano A, Settineri N, Ascenti G, Blandino A (2011) Muscle fat fraction in neuromuscular disorders: dual-echo dual-flip-angle spoiled gradient-recalled $\mathrm{mr}$ imaging technique for quantification—a feasibility study. Radiology 259:487-494. doi:10.1148/ radiol.10101108

14. Meola G, Bugiardini E, Cardani R (2011) Muscle biopsy. J Neurol. doi:10.1007/s00415-011-6193-8

15. Huang Y, Majumdar S, Genant HK, Chan WP, Sharma KR, Yu P, Mynhier M, Miller RG (1994) Quantitative MR relaxometry study of muscle composition and function in Duchenne muscular dystrophy. JMRI 4:59-64

16. Patten C, Meyer RA, Fleckenstein JL (2003) T2 mapping of muscle. Semin Musculoskel Radiol 7:297-305. doi:10.1055/ s-2004-815677

17. Skinner TE, Glover GH (1997) An extended two-point Dixon algorithm for calculating separate water, fat, and B0 images. Magn Reson Med 37:628-630

18. Allerhand A (1966) Analysis of carr-purcell spin-echo nmr experiments on multiple-spin systems. I. the effect of homonuclear coupling. J Chem Phys 44:1-9 